\title{
Neonatal outcome of small for gestational age infants born at 26-33 weeks' gestation in Chinese neonatal intensive care units
}

\author{
Yihuang Huang ${ }^{1 \#}$, Lan Zhang ${ }^{1 \#}$, Huiqing Sun ${ }^{2}$, Cuiqing Liu ${ }^{3,4}$, Yi Yang ${ }^{5}$, Shoo K. Lee ${ }^{6,7,8}$, Yun Cao ${ }^{1}$, \\ Siyuan Jiang ${ }^{1}$; on behalf of the Reduction of Infection in Neonatal Intensive Care Units using the \\ Evidence-based Practice for Improving Quality (REIN-EPIQ) Study Group
}

${ }^{1}$ Department of Neonatology, Children's Hospital of Fudan University, Shanghai, China; ${ }^{2}$ Department of Neonatology, Henan Children's Hospital, Zhengzhou, China; ${ }^{3}$ Department of Neonatology, Hebei Children's Hospital, Shijiazhuang, China; ${ }^{4}$ Department of Neonatology, Sanya People's Hospital, Sanya, China; ${ }^{5}$ NHC Key Laboratory of Neonatal Diseases (Fudan University), Children's Hospital of Fudan University, Shanghai, China; ${ }^{6}$ Maternal-Infant Care Research Centre and Department of Pediatrics, Mount Sinai Hospital, Toronto, Canada; ${ }^{7}$ Department of Pediatrics, University of Toronto, Toronto, Canada; ${ }^{8}$ Department of Obstetrics and Gynecology and Dalla Lana School of Public Health, University of Toronto, Toronto, Canada

Contributions: (I) Conception and design: SK Lee, S Jiang, Y Cao; (II) Administrative support: SK Lee, Y Cao; (III) Provision of study materials or patients: S Jiang, Y Huang, L Zhang, SK Lee, Y Cao; (IV) Collection and assembly of data: L Zhang, H Sun, C Liu; (V) Data analysis and interpretation: S Jiang, Y Huang; (VI) Manuscript writing: All authors; (VII) Final approval of manuscript: All authors.

\#These authors contributed equally to this work.

Correspondence to: Yun Cao, Siyuan Jiang. Department of Neonatology, Children's Hospital of Fudan University, 399 Wanyuan Road, Minhang District, Shanghai 201102, China. Email: yuncao@fudan.edu.cn; jiangsiyuan@fudan.edu.cn.

Background: Rate and outcomes of small for gestational age (SGA) infants admitted to Chinese neonatal intensive care units (NICU) has been poorly demonstrated. We aimed to describe the rate and outcomes of SGA preterm infants in Chinese NICU, and to evaluate the association of SGA status with neonatal outcomes in different gestational age (GA) and birth weight percentile groups.

Methods: This cohort study included all infants born at 26-33 weeks' gestation and admitted to 25 tertiary Chinese NICUs from April 2015 to May 2018. SGA was defined as a birthweight <10th percentile for GA based on the Chinese neonatal birth weight curve.

Results: A total of 24,596 infants were included, and 1,867 (7.6\%) infants were SGA. SGA infants had significantly higher rates of death or any major morbidity $(29.8 \%$ vs. $20.5 \%)$, mortality $(7.0 \%$ vs. $4.1 \%)$, bronchopulmonary dysplasia (BPD, 17.6\% vs. 9.8\%), necrotizing enterocolitis (NEC, $4.8 \%$ vs. 3.2\%) and sepsis $(7.3 \%$ vs. $4.8 \%)$ than non-SGA infants. SGA status was independently associated with increased risk of death or any major morbidity [adjusted odds ratio: 2.37 (2.08-2.71)] as well as increased risks of death, BPD, ROP, death or BPD, death or ROP, NEC and sepsis. The increased risks of adverse outcomes for SGA infants existed across GA groups. The risks of adverse outcomes were highest among infants with a birthweight $<3$ rd percentile.

Conclusions: SGA contributes significantly to adverse neonatal outcomes. Specific attentions are warranted when caring for SGA preterm infants.

Keywords: Small for gestational age (SGA); preterm; neonatal outcome; China

Submitted Oct 14, 2020. Accepted for publication Feb 15, 2021.

doi: $10.21037 / \mathrm{tp}-20-339$

View this article at: http://dx.doi.org/10.21037/tp-20-339 


\section{Introduction}

Small for gestational age (SGA) status, which is defined as a birthweight $<$ the 10th percentile, has long been considered a vital risk factor for neonatal mortality and morbidities among preterm infants $(1,2)$. Increased risks of bronchopulmonary dysplasia (BPD) (3), late-onset sepsis (4), necrotizing enterocolitis (NEC) (5), retinopathy of prematurity (ROP) (6) and death (5) have been observed among SGA infants compared to non-SGA infants in previous studies. Therefore, SGA preterm infants require extra attention during their stay in neonatal intensive care units (NICUs). With the advancement of perinatal medicine, an increased number of preterm infants have survived in China (7). However, the rate of SGA and the risks of neonatal mortality and morbidities for SGA preterm infants in Chinese NICUs have been scarcely demonstrated.

This study aimed to estimate the rate and neonatal outcomes of SGA preterm infants in the largest contemporary multicenter cohort of preterm infants in China. We also evaluated the association of SGA status with neonatal outcomes in different GA and birth weight percentile groups, aiming to demonstrate whether the association existed among different GA groups and birth weight percentile groups.

We present the study in accordance with the STROBE reporting checklist (available at http://dx.doi.org/10.21037/ tp-20-339).

\section{Methods}

The study was conducted in accordance with the Declaration of Helsinki (as revised in 2013). The study was approved by The Ethics Committee of the Children's Hospital of Fudan University (Ethic approval No. [2015] 28) and informed consent was waived.

\section{Study design and population}

This is a cohort study of preterm infants admitted to 25 tertiary hospitals across China that used prospectively collected data in a standardized clinical database. The clinical database was initially established for a cluster randomized controlled trial "Reduction of Infection in NICUs using the Evidence-based Practice for Improving Quality (EPIQ)" (REIN-EPIQ study, clinicaltrials.gov \#NCT02600195) (8). Twenty-five hospitals continuously collected clinical data of all admitted preterm infants
$<34$ weeks' gestation for three years (May 1st, 2015 to April 30th, 2018). All data collection processes followed standard operating procedures and definitions (7).

The inclusion criteria of this study were all infants born at $26^{+0}-33^{+6}$ weeks' gestation and admitted to participating hospitals within 7 days after birth from May 1st, 2015 to April 30th, 2018. Stillborn infants and infants who died in the delivery room were not eligible. Infants who did not receive complete care and left the hospital against medical advice were all excluded. Infants were followed until death or discharge from the NICU.

\section{Setting}

Twenty-five tertiary hospitals from 19 provinces in China participated in this study. All hospitals were able to provide comprehensive care for infants born $<28$ weeks of gestation or weighing $<1,000$ grams at birth. Among the participating hospitals, 17 were national or provincial neonatal referral centers, 8 were regional referral centers in metropolitan cities, 18 were perinatal centers, and 7 were specialized children's hospitals. Twelve hospitals were located in eastern region of China, nine hospitals in central region and the other four in western China (7).

\section{Definitions}

SGA status was defined as a birthweight $<$ the 10 th percentile for gestational age according to the Chinese neonatal birth weight curve for gestational age published in 2015 (9). Gestational age (GA) was determined using the hierarchy of best obstetric estimate based on the prenatal ultrasound, menstrual history, obstetric examination, or all three. Antenatal corticosteroid therapy was defined as intravenous glucocorticoid treatment in one or more doses before delivery. The Transport Risk Index of Physiologic Stability (TRIPS) score was used as an illness severity score on NICU admission (10).

The primary outcomes were neonatal mortality and any major morbidity, including BPD, intraventricular hemorrhage $(\mathrm{IVH}) \geq$ grade III or periventricular leukomalacia (PVL), NEC, ROP and sepsis. BPD was defined as the need for mechanical ventilation or oxygen dependency at 36 weeks of postmenstrual age or at discharge (11). NEC was defined as $\geq$ stage 2 according to the Bell criteria $(12,13)$. Sepsis was defined by a positive blood or cerebral spinal fluid culture. Cultures that were positive for coagulase-negative staphylococcus (CONS) 


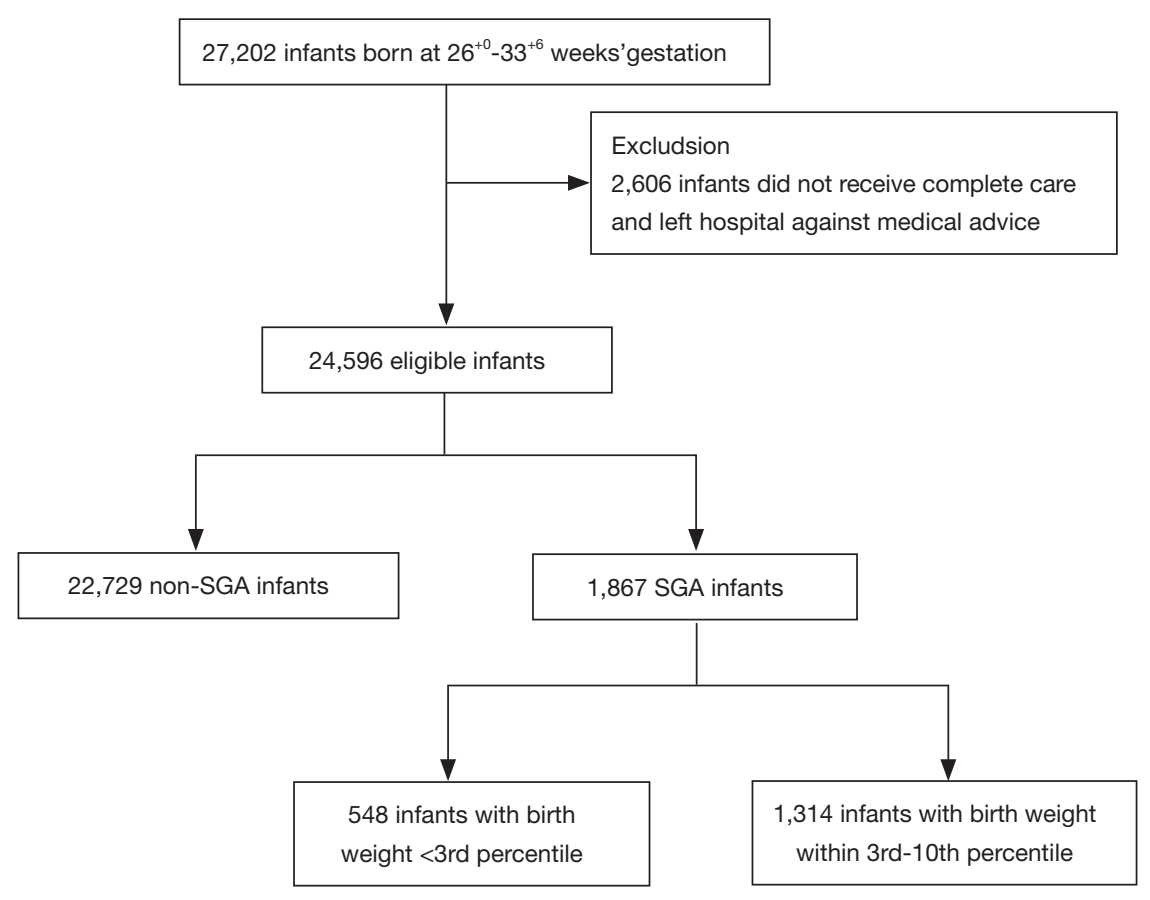

Figure 1 Study population.

were considered as definite or possible infection if the infants fulfilled all three criteria below: (I) infection-related clinical manifestations; (II) two positive cultures drawn within two days of each other, or one positive blood culture together with abnormal white blood cell count $\left(<5 \times 10^{6} / \mathrm{L}\right.$ or $\left.>20 \times 10^{6} / \mathrm{L}\right)$, C-reactive protein level $(\geq 8 \mathrm{mg} / \mathrm{L})$, or procalcitonin level $(>0.5 \mathrm{ng} / \mathrm{mL})$ within two days (14) susceptible antibiotics were given or planned $\geq 5$ days (15). IVH was classified according to Papile criteria (16). PVL was defined as the presence of periventricular cysts on cranial ultrasound or cranial MRI scans before discharge. ROP was diagnosed according to the International Classification of ROP (17).

\section{Statistical analysis}

Student's $t$-tests and Pearson $\chi^{2}$ tests were used to compare the distributions of continuous variables and categorical variables between the SGA and non-SGA groups, respectively. Multilevel mixed-effects logistic regression models were used to examine the association between SGA and neonatal outcomes, accounting for the intracluster correlation among the infants within hospitals. Hospitals were considered independent clusters with random effects in the models. At the infant level, gestational age, sex, Apgar scores $<3$ at 1 and 5 minutes, congenital abnormalities, TRIPS score on admission, cesarean status, inborn status, primigravida, prenatal care, maternal hypertension, maternal diabetes, and antenatal steroids were adjustment factors. Same analyses were performed for infants with different GAs and with birth weights < the 3rd percentile and within the $3 \mathrm{rd}-10$ th percentiles. In all analyses, a twosided $\mathrm{P}<0.05$ was used to determine statistical significance. Statistical analysis was performed using Stata 15.1 (StataCorp, College Station, TX).

\section{Results}

\section{Infant and maternal characteristics}

Overall, 27,202 infants with gestational age from 26 to 33 weeks were admitted to participating NICUs during the study period. After excluding 2,606 infants with incomplete care, this study included a total of 24,596 infants, among whom 1,867 (7.6\%) were SGA (shown in Figure 1). Among all SGA preterm infants, $548(2.2 \%)$ had a birth weight < the $3^{\text {rd }}$ percentile, and $1,314(5.3 \%)$ within the $3 \mathrm{rd}-10$ th percentiles.

The infant and maternal characteristics stratified by SGA status are described in Table 1. SGA preterm infants had lower Apgar scores at 1 minute after birth and higher 
Table 1 Infant and Maternal Characteristics for SGA or non-SGA infants born at 26-33 weeks

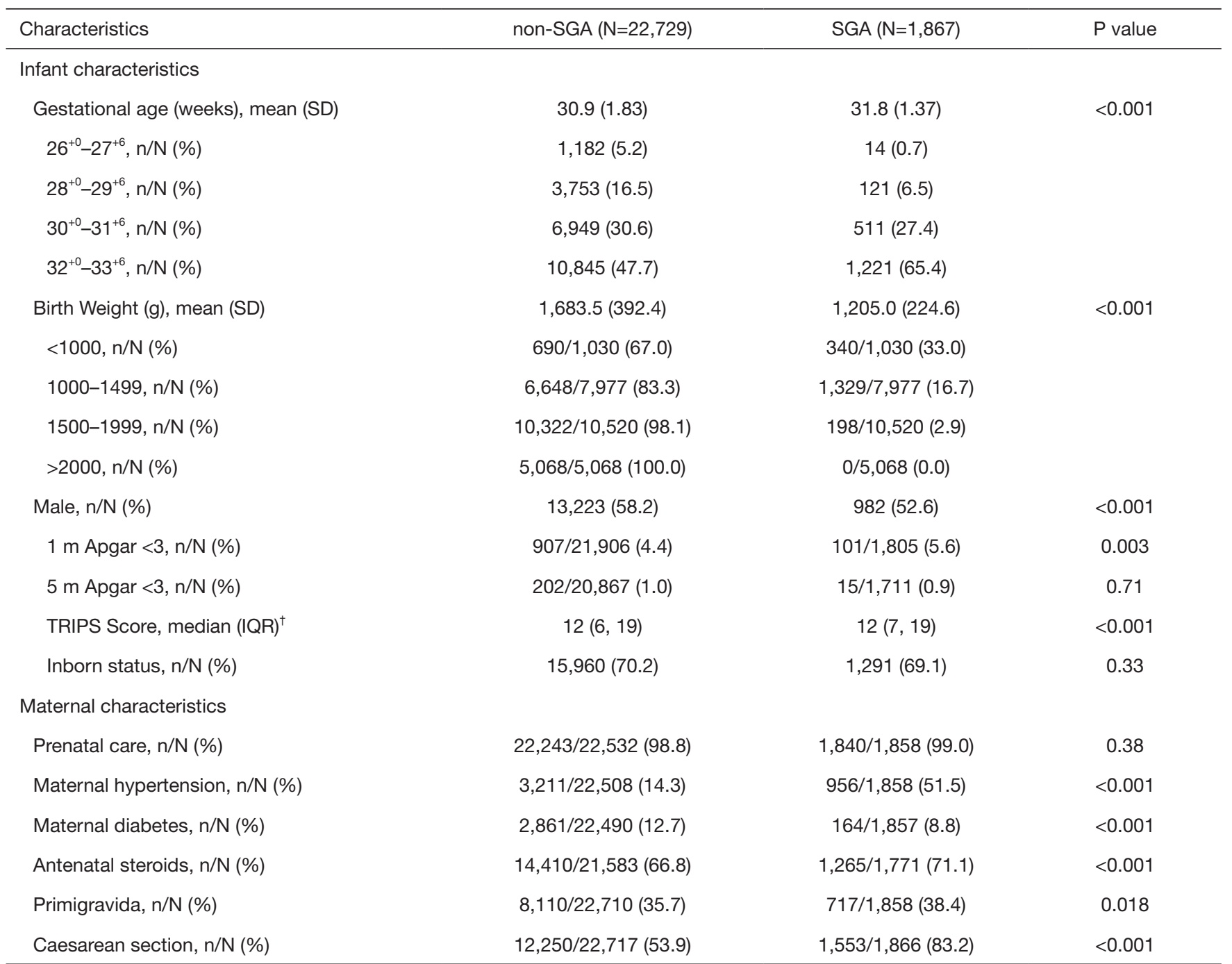

${ }^{\dagger}$, Data on TRIPS Score were missing in 114 infants. SGA, small for gestational age; SD, standard deviation. TRIPS Score, cesarean, congenital anomalies, inborn status, primigravida, prenatal care, maternal hypertension, maternal diabetes and antenatal steroids were adjusted.

TRIPS scores on admission than non-SGA preterm infants. Mothers of SGA preterm infants were more likely to be primigravida ( $38.4 \%$ vs. $35.7 \%, \mathrm{P}=0.018$ ), to have hypertension $(51.5 \%$ vs. $14.3 \%, \mathrm{P}<0.001)$, to receive antenatal steroids $(71.1 \%$ vs. $66.8 \%, \mathrm{P}<0.001)$ and to deliver by cesarean section $(83.2 \%$ vs. $53.9 \%, \mathrm{P}<0.001)$ but were less likely to have diabetes $(8.8 \%$ vs. $12.7 \%, \mathrm{P}<0.001)$.

\section{Neonatal outcomes}

The rate of death or any morbidity for SGA preterm infants was significantly higher than that for non-SGA preterm infants $(29.8 \%$ vs. $20.5 \%, \mathrm{P}<0.001)$. Concerning each outcome, the mortality rate $(7.0 \%$ vs. $4.1 \%, \mathrm{P}<0.001)$ and incidences of $\mathrm{BPD}(17.6 \%$ vs. $9.8 \%, \mathrm{P}<0.001)$, death or BPD (19.8\% vs. $11.1 \%, \mathrm{P}<0.001)$, death or ROP $(17.7 \%$ vs. $12.9 \%, \mathrm{P}<0.001)$, NEC ( $4.8 \%$ vs. $3.2 \%, \mathrm{P}<0.001)$ and sepsis (7.3\% vs. $4.8 \%, \mathrm{P}<0.001)$ were all remarkably higher among SGA preterm infants than non-SGA preterm infants (shown in Table 2).

After adjustment, SGA preterm infants had a 2.37 fold (95\% CI: 2.08-2.71) increased risk of death or any morbidity compared with non-SGA preterm infants. SGA was also independently associated with increased risks of 
Table 2 Neonatal outcomes for SGA or non-SGA infants born at 26-33 weeks

\begin{tabular}{|c|c|c|c|c|c|c|}
\hline Outcomes & non-SGA $(\mathrm{N}=22,732)$ & \multicolumn{3}{|c|}{ SGA } & $P$ value* & $P$ value $e^{\star \star}$ \\
\hline Death, n/N (\%) & $926(4.1)$ & $131(7.0)$ & $48(8.8)$ & $83(6.3)$ & $<0.001$ & $<0.001$ \\
\hline $\mathrm{ROP}^{\ddagger}, \mathrm{n} / \mathrm{N}(\%)$ & 2,006/12,107 (16.6) & 202/1,326 (15.2) & 66/405 (12.0) & 137/921 (10.4) & 0.21 & 0.007 \\
\hline Death or BPD, n/N (\%) & $2,532(11.1)$ & $370(19.8)$ & $142(25.9)$ & $228(17.3)$ & $<0.001$ & $<0.001$ \\
\hline Death or ROP, n/N (\%) & 2,927 (12.9) & $331(17.7)$ & $113(20.6)$ & $218(16.5)$ & $<0.001$ & $<0.001$ \\
\hline $\begin{array}{l}\mathrm{IVH} \geq \text { grade III or } \mathrm{PVL}^{\dagger} \\
\mathrm{n} / \mathrm{N}(\%)\end{array}$ & 1,238/20,932 (5.9) & $100 / 1,732(5.8)$ & $30 / 454(6.6)$ & $70 / 1,278$ (5.5) & 0.81 & 0.66 \\
\hline Sepsis, n/N (\%) & $1,094(4.8)$ & $137(7.3)$ & $43(8.6)$ & $94(6.9)$ & $<0.001$ & $<0.001$ \\
\hline \multicolumn{7}{|c|}{$\begin{array}{l}{ }^{*} \text {, Comparison among non-SGA and SGA infants; }{ }^{* *} \text {, Comparison among non-SGA infants, infants with birth weight }<3^{\text {rd }} \text { percentile and } \\
\text { 3rd-10th percentile. }{ }^{\dagger} \text {, Incidence of PVL was calculated among infants with neuroimaging results; }{ }^{\ddagger} \text {, Incidence of ROP } \geq \text { stage } 3 \text { was } \\
\text { calculated among infants with eye examinations in the NICU. SGA, small for gestational age; BPD, bronchopulmonary dysplasia; ROP, } \\
\text { retinopathy of prematurity; IVH, intraventricular hemorrhage; PVL, periventricular leukomalacia; NEC, necrotizing enterocolitis; NICU, } \\
\text { neonatal intensive care unit. }\end{array}$} \\
\hline
\end{tabular}

death, BPD, ROP, death or BPD, death or ROP, NEC and sepsis (shown in Figure 2 and Table S1).

Among infants with different GAs, we found that the increased risks of adverse outcomes for SGA infants existed across GA groups. The incidences of death or any morbidity were higher for SGA infants at each gestational week $\geq 29$ weeks. Increased mortality rates and BPD incidences were observed for SGA infants in each GA group. The incidences of ROP and NEC were significantly higher among SGA infants $\geq 30$ weeks (shown in Figure 3 and Table S2). The association of SGA status and adverse outcomes across GA groups remained similar after adjustment (shown in Figure S1).

For infants with birth weight $<$ the 3 rd percentile and within the 3rd-10th percentiles, we found that the risks of adverse outcomes increased as the birth weight percentile decreased (shown in Figure 2 and Table S3). Infants with a birth weight within the 3rd-10th percentiles were exposed to notably increased risks of death or any morbidity, including BPD, ROP, death or BPD, death or ROP, and sepsis, compared with non-SGA preterm infants; moreover, infants with a birth weight $<$ the 3 rd percentile had the highest risks among the three groups.

\section{Care practices}

Care practices in NICUs for SGA or non-SGA preterm infants are shown in Table 3. SGA preterm infants were more likely to receive surfactant $(29.7 \%$ vs. $25.7 \%$, $\mathrm{P}=0.002)$, central venous lines $(64.8 \%$ vs. $35.5 \%, \mathrm{P}<0.001)$ and vasopressors $(22.5 \%$ vs. $17.2 \%, \mathrm{P}<0.001)$ than non-SGA preterm infants. Oral feeds were initiated later for SGA patients, even though a larger proportion of SGA preterm infants were fed breastmilk. In addition, SGA preterm infants were more likely to receive parenteral nutrition (94.2\% vs. $91.0 \%, \mathrm{P}<0.001)$ and have a longer hospital stay. Care practices among different GA groups presented similar trends (shown in Table S4).

\section{Discussion}

Neonatal outcomes for SGA preterm infants among 24,596 preterm newborns admitted to 25 NICUs from 19 provinces were presented in this study, which, to the best of our knowledge, has the largest sample of preterm infants in China to date. We identified significantly higher risks of mortality and major morbidities for SGA preterm infants compared with those for non-SGA preterm infants, as well 


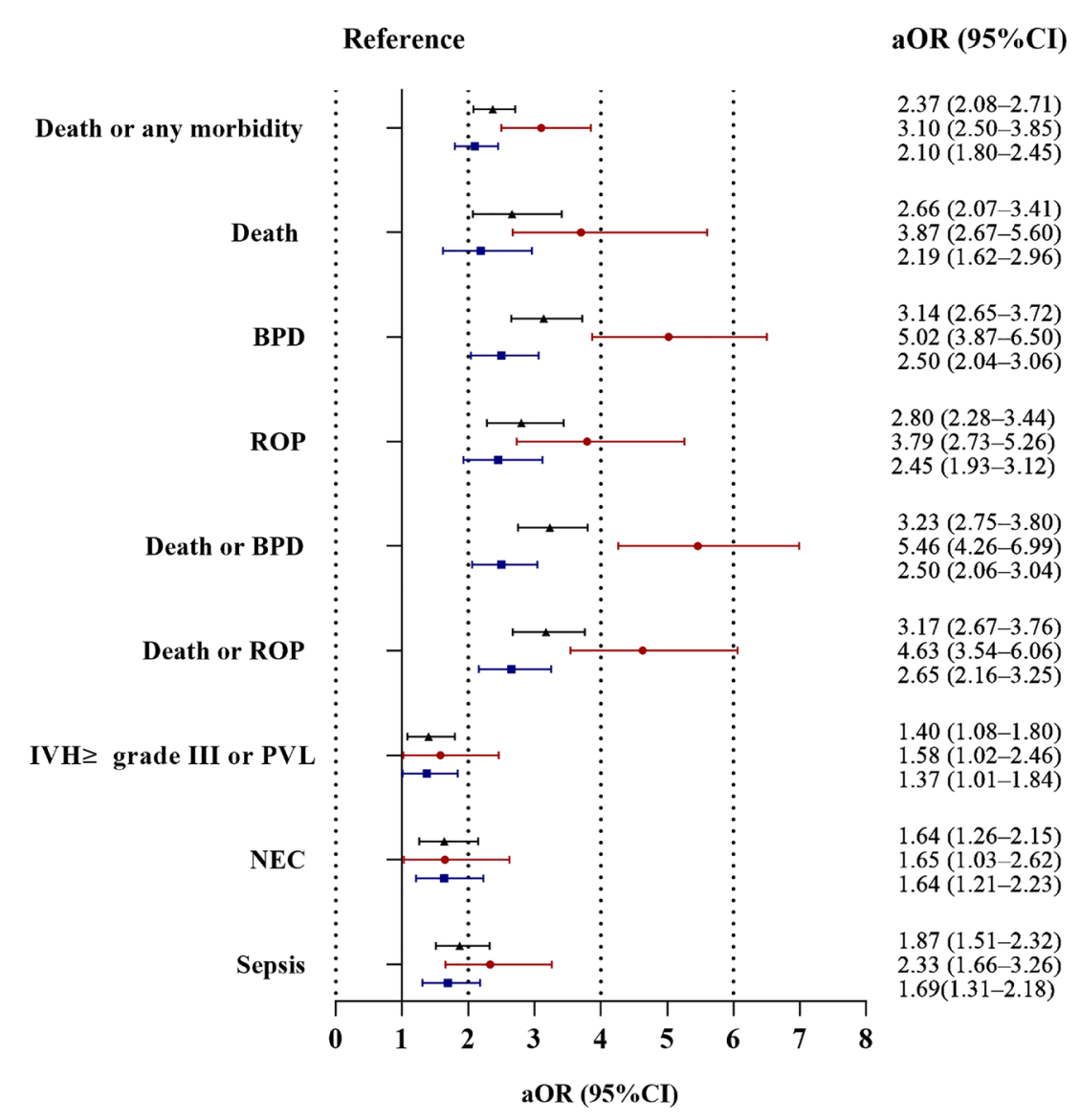

- All SGA infants (birth weight $<10$ th percentile)

- Birth weight $<3$ rd percentile

- Birth weight within 3rd-10th percentile

Figure 2 Risks of mortality and major morbidities among all SGA infants and infants with birth weight <3rd percentile and within 3rd-10th percentile. Multilevel mixed-effects logistic regression models were used accounting for the correlations among infants within hospitals. At the infant level, gestational age, sex, Apgar score $<3$ at 1 and 5 minutes, TRIPS Score, cesarean, congenital anomalies, inborn status, primigravida, prenatal care, maternal hypertension, maternal diabetes and antenatal steroids were adjusted. BPD, bronchopulmonary dysplasia; ROP, retinopathy of prematurity; IVH, intraventricular hemorrhage; PVL, periventricular leukomalacia; NEC, necrotizing enterocolitis; aOR, adjusted odds ratio; SGA, small for gestational age; TRIPS Score, cesarean, congenital anomalies, inborn status, primigravida, prenatal care, maternal hypertension, maternal diabetes and antenatal steroids were adjusted.

as an increased requirement for NICU resources among SGA preterm infants.

Among 24,596 infants born at 26-33 weeks' gestation and admitted to Chinese NICUs, 1,867 (7.6\%) were classified as SGA. This SGA rate for preterm infants was lower than that reported previously, which varied from $9.95 \%$ to $37.5 \%$ in developed countries including the US (5), Canada (18), Sweden (19), Italy (20) and Germany (21). In addition, the SGA rate was also lower than we expected, that the rate might be approximately
$10 \%$ based on the definition of SGA. We suspected that this relatively low SGA rate was because our study was hospitalbased instead of birth-population-based. For infants $>32$ weeks of gestation, those in good condition would not be admitted to tertiary NICUs. However, more importantly, we observed a decreased proportion of SGA infants with decreasing GA, and specifically, only $3.1 \%$ of infants born at 28-29 weeks gestation and $1.2 \%$ of infants born at 26-27 weeks' gestation were SGA. This result indicated that many SGA infants at the youngest GA or with 

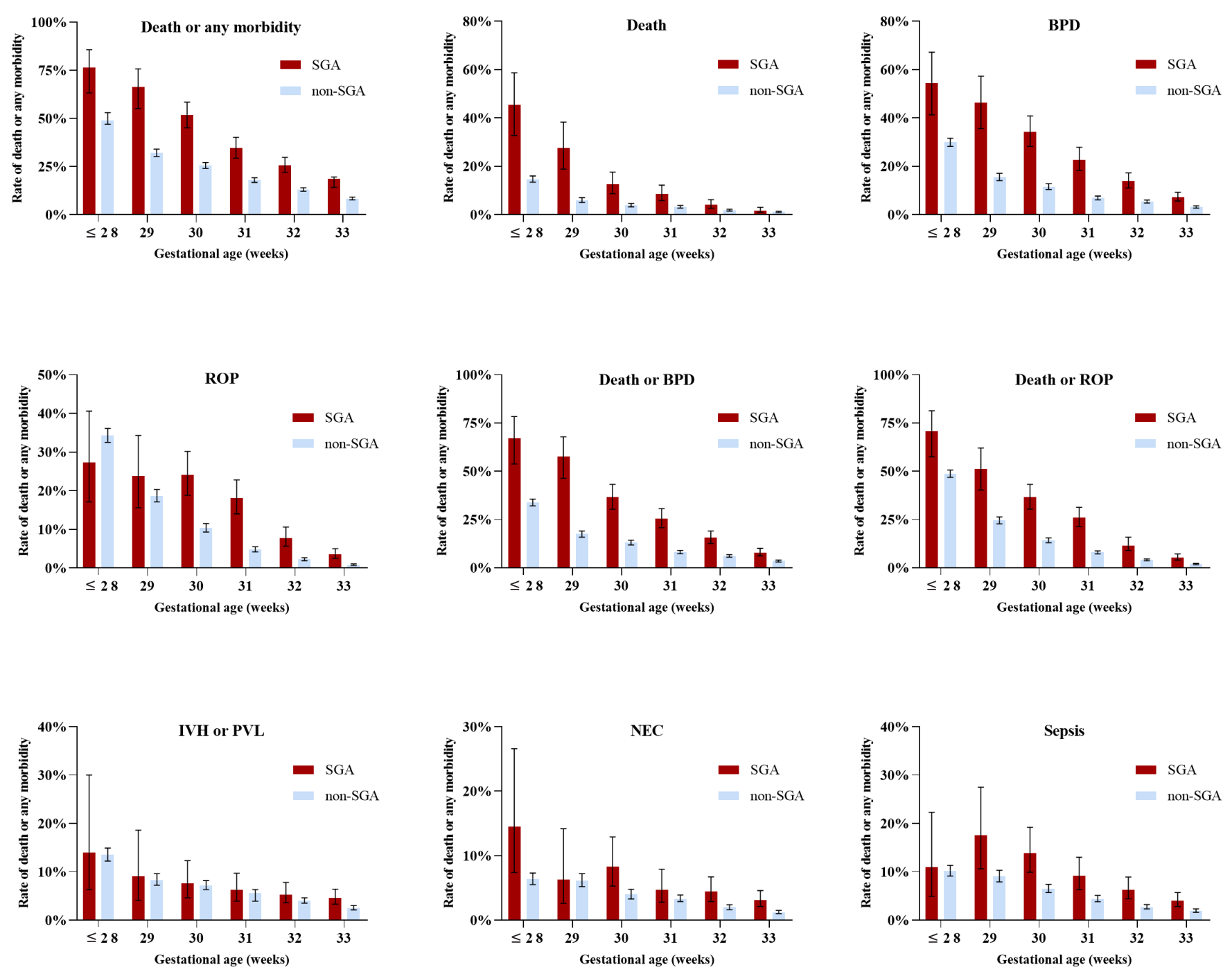

Figure 3 Rates of mortality and major morbidities for SGA and non-SGA infants by gestational age. SGA, small for gestational age; BPD, bronchopulmonary dysplasia; ROP, retinopathy of prematurity; IVH, intraventricular hemorrhage; PVL, periventricular leukomalacia; NEC, necrotizing enterocolitis.

highest illness severity might die in the delivery room or receive palliative care without NICU admission. More investigations are needed for the exact reasons and to facilitate possible quality improvement initiatives.

Based on our results, SGA status contributed greatly to neonatal death. The risk of death was 2.66-fold higher for SGA preterm infants compared with non-SGA preterm infants after adjustment. The increased risk of death was most significant among infants with the lowest gestational age. However, even among infants born $\geq 30$ weeks gestation, who presented a relatively low mortality rate, the mortality rate of SGA preterm infants was substantial. Due to the paucity of information on the causes of death, a detailed explanation for such a high incidence and risk of death could not be further inferred, but these data are required in future studies to develop specific approaches for reducing the mortality rate.

We identified a 3.1-fold higher risk of BPD among SGA preterm infants compared with non-SGA preterm infants, which was in accordance with previous studies that have reported a 2-6-fold higher risk of BPD for SGA preterm infants $(3,22,23)$. A large proportion of SGA preterm 
Table 3 Care practice in NICUs for SGA and non-SGA infants born at 26-33 weeks

\begin{tabular}{|c|c|c|c|}
\hline Care practice & non-SGA $(\mathrm{N}=22,729)$ & SGA $(N=1,867)$ & $P$ value \\
\hline Mechanical ventilation, n/N (\%) & $5,939(26.1)$ & $458(24.5)$ & 0.13 \\
\hline Duration of invasive ventilation, median [IQR] & $4[2,7]$ & $4[2,8]$ & 0.061 \\
\hline Non-Invasive Ventilation, n/N (\%) & $13,925(61.3)$ & $1,150(61.6)$ & 0.78 \\
\hline Central Venous Line, n/N (\%) & $8,077(35.5)$ & $1,210(64.8)$ & $<0.001$ \\
\hline Duration of Central Venous Line, median [IQR] & $20[11,30]$ & $21[13,31]$ & 0.005 \\
\hline Vasopressors use, n/N (\%) & $3,913(17.2)$ & $420(22.5)$ & $<0.001$ \\
\hline Duration of vasopressors use, median [IQR] & $4[2,7]$ & $4[2,8]$ & 0.62 \\
\hline Duration of Parenteral Nutrition, median [IQR] & $14[9,23]$ & $20[13,30]$ & $<0.001$ \\
\hline 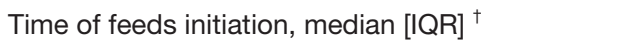 & $2[2,3]$ & $2[2,3]$ & $<0.001$ \\
\hline$<24 \mathrm{~h}$ & $4,872 / 21,431(22.7)$ & 290/1,793 (16.7) & $<0.001$ \\
\hline $24-48$ h & $9,733 / 21,431(45.4)$ & $811 / 1,793$ (46.8) & \\
\hline $48-72 \mathrm{~h}$ & $3,065 / 21,431(14.3)$ & 240/1,793 (13.8) & \\
\hline$>72 \mathrm{~h}$ & $3,761 / 21,431(17.5)$ & $393 / 1.793(22.7)$ & \\
\hline Breastmilk feeding, n/N (\%) & $12,383(54.5)$ & $1,064(57.0)$ & 0.036 \\
\hline NICU stay (days), median [IQR $]^{\ddagger}$ & $27[17,41]$ & $39[29,53]$ & $<0.001$ \\
\hline
\end{tabular}

${ }^{\dagger}$, Days of feeds initiation were missing in 1,372 infants; ${ }^{\ddagger}$, Infants who died in the NICU were excluded. NICU, neonatal intensive care unit; SGA, small for gestational age; IQR, interquartile range, PMA, post menstrual age.

infants may also experience intrauterine growth restriction (IUGR), one of the major risk factors for BPD. A reduced postnatal pulmonary diffusing capacity, mild hypoxemia, increased metabolic rate, changes in lung structure and lung and chest dysfunction all contributed to the mechanisms of permanent respiratory deficiency in IUGR infants in animal studies $(24,25)$.

It was observed that the risk of ROP was significantly increased for SGA infants $\geq 30$ weeks gestation. As mentioned above, SGA infants were at higher risk of respiratory disorders and thus were associated with a higher rate and longer duration of oxygen supplementation, which was a significant risk factor for ROP development (26). A systematic review included 21 studies and evaluated the association between ROP and SGA in preterm infants. The results reported increased ROP odds for SGA infants among all preterm infants (6). The small sample size of infants with the youngest gestational age have limited our power to detect differences in ROP incidence among infants $\leq 29$ weeks' gestation. Furthermore, $35 \%$ of SGA infants with a GA $\leq 29$ weeks died, and these deaths may have occurred before ROP could develop. Our results indicated that SGA preterm infants, even among those with relatively larger GAs, should be more closely monitored for ROP. In light of the increased risks of BPD and ROP among SGA infants, it might worth to evaluate the effects of relevant care practices, such as saturation targets, specifically in the SGA preterm population to guide more precise care for these infants.

The risks of NEC were higher in SGA infants born at $\geq 30$ weeks gestation. This result agreed with previously published results $(5,27,28)$. For feeding practices, although the breastfeeding rate was higher among SGA preterm infants, SGA preterm infants still had a higher rate and 
longer duration of parenteral nutrition and started oral feeding later than non-SGA preterm patients. This result indicated that feeding tolerance might be worse in SGA preterm infants, which might be related to the increased risk of NEC.

The association between SGA and sepsis was also demonstrated in our study and previous reports $(4,29)$. The potential mechanism may be associated with the immaturity of SGA preterm infants, longer stay in the NICU and more invasive care practices, leading to a higher probability of infection.

In this study, we elaborated on the rates and risks of neonatal outcomes for SGA preterm infants based on the largest contemporary cohort of preterm infants in China, thus providing useful references for neonatologists. There were also several limitations. First, this was a hospital-based study, and the proportion of SGA preterm infants could not represent the incidence of SGA status in the population. There might be a considerable proportion of SGA infants with the lowest gestational age did not receive active care and were not admitted to NICUs. Second, although the database provided detailed definitions, we were unable to evaluate potential diagnostic variabilities across sites (e.g., differences in timing and diagnostic criteria of cranial ultrasounds or ophthalmologic examinations). Third, the sample size of infants born $<28$ gestational weeks was relatively small and did not have enough statistical power. Fourth, there was no information on the causes of SGA status in our study, and these causes may be closely associated with neonatal outcomes. Fifth, 2,606 infants were excluded in our analysis due to incomplete care (discharge against medical advice) and unpredictable outcomes. Among these infants, 11.0\% $(287 / 2,606)$ were SGA and therefore the incidence of SGA presented in our study was mildly underestimated.

In conclusion, $7.6 \%$ of infants born at 26-33 weeks and admitted to Chinese NICUs were SGA. SGA is significantly associated with adverse neonatal outcomes across GA groups. Specific attention for SGA preterm infants in NICUs and better perinatal monitoring and management to reduce the SGA rate are both required.

\section{Acknowledgments}

The REIN-EPIQ Study Group: Yun Cao, Siyuan Jiang, Children's Hospital of Fudan Univeristy; Yong Ji, Children's Hospital of ShanXi / Women's Health Center of Shanxi, Shanxi, China; Shuping Han, Women's Hospital of Nanjing Medical University, Jiangsu, China; Sannan Wang, Suzhou
Municipal Hospital, Jiangsu, China; Zhankui Li, Northwest Women and Children's Hospital, Shaanxi, China; Shiwen Xia, Women and Children's Hospital of Hubei Province, Hubei, China; Changyi Yang, Fujian Provincial Maternity and Children's Hospital/Affiliated Hospital of Fujian Medical University, Fujian, China; Chuanzhong Yang, The Affiliated Shenzhen Maternity and Child Healthcare Hospital of Southern Medical University, Guangdong, China; Ling Chen, Tongji Hospital, Tongji Medical College, Huazhong University of Science and Technology, Hubei, China; Ruobing Shan, Qingdao Women and Children's Hospital, Shandong, China; Ling Liu, Guiyang Maternal and Child Health Care Hospital, Guizhou, China; Bin Yi, Gansu Provincial Maternity and Child-care Hospital, Gansu, China; Zhenlang Lin, The $2^{\text {nd }}$ Affiliated Hospital and Yuying Children's Hospital of Wenzhou Medical University, Zhejiang, China;Yang Wang, The First Affiliated Hospital of Anhui Medical University, Anhui, China; Jiangqin Liu, Shanghai First Maternity and Infant Hospital, Tongji University School of Medicine, Shanghai, China; Ling He, Jiangxi Provincial Children's Hospital, Jiangxi, China; Mingxia Li, MD, First Affiliated Hospital of Xinjiang Medical University, Xinjiang, China; Xinnian Pan, The Maternal and Child Health Hospital of Guangxi Zhuang Autonomous Region, Guangxi, China; Yan Guo, Children's Hospital of Nanjing Medical University, Jiangsu, China; Cuiqing Liu, Children's Hospital of Hebei Province, Hebei, China; Qin Zhou, The Affiliated Wuxi Maternity and Child Health Hospital of Nanjing Medical University, Jiangsu, China; Xiaoying Li, Qilu Children's Hospital of Shandong University, Shandong, China; Hong Xiong, Children's Hospital Affiliated to Zhengzhou University, Henan, China; Yujie Qi, Beijing Children's Hospital of Capital Medical University, Beijing, China; Mingyan Hei, The Third Xiangya Hospital of Central South University, Hunan, China.

We would like to thank American Journal Expert for its help in polishing the language of our paper.

Funding: This study was funded by the China Medical Board (Grant Number 14-194 [to YC]) and the Canadian Institutes of Health Research (Grant Number CTP 87518 [to SKL]).

\section{Footnote}

Reporting Checklist: The authors have completed the STROBE reporting checklist. Available at http://dx.doi. org/10.21037/tp-20-339

Data Sharing Statement: Available at http://dx.doi. 
org/10.21037/tp-20-339

Conflicts of Interest: All authors have completed the ICMJE uniform disclosure form (available at http://dx.doi. org/10.21037/tp-20-339). The authors have no conflicts of interest to declare.

Ethical Statement: The authors are accountable for all aspects of the work in ensuring that questions related to the accuracy or integrity of any part of the work are appropriately investigated and resolved. The study was conducted in accordance with the Declaration of Helsinki (as revised in 2013). The study was approved by The Ethics Committee of the Children's Hospital of Fudan University (Ethic approval No. [2015] 28) and informed consent was waived.

Open Access Statement: This is an Open Access article distributed in accordance with the Creative Commons Attribution-NonCommercial-NoDerivs 4.0 International License (CC BY-NC-ND 4.0), which permits the noncommercial replication and distribution of the article with the strict proviso that no changes or edits are made and the original work is properly cited (including links to both the formal publication through the relevant DOI and the license). See: https://creativecommons.org/licenses/by-nc-nd/4.0/.

\section{References}

1. Bernstein IM, Horbar JD, Badger GJ, et al. Morbidity and mortality among very-low-birth-weight neonates with intrauterine growth restriction. The Vermont Oxford Network. Am J Obstet Gynecol 2000;182:198-206.

2. De Jesus LC, Pappas A, Shankaran S, et al. Outcomes of small for gestational age infants born at $<27$ weeks' gestation. J Pediatr 2013;163:55-60.e603.

3. Rocha G, de Lima FF, Machado AP, et al. Small for gestational age very preterm infants present a higher risk of developing bronchopulmonary dysplasia. J Neonatal Perinatal Med 2019;12:419-27.

4. Tröger B, Göpel W, Faust K, et al. Risk for late-onset blood-culture proven sepsis in very-low-birth weight infants born small for gestational age: a large multicenter study from the German Neonatal Network. Pediatr Infect Dis J 2014;33:238-43.

5. Boghossian NS, Geraci M, Edwards EM, et al. Morbidity and Mortality in Small for Gestational Age Infants at 22 to 29 Weeks' Gestation. Pediatrics 2018;141:e20172533.
6. Razak A, Faden M. Association of small for gestational age with retinopathy of prematurity: a systematic review and meta-analysis. Arch Dis Child Fetal Neonatal Ed 2020;105:270-8.

7. Jiang S, Yan W, Li S, et al. Mortality and Morbidity in Infants <34 Weeks' Gestation in 25 NICUs in China: A Prospective Cohort Study. Front Pediatr 2020;8:33.

8. REIN-EPIQ Study Group Reduction of Infection in Neonatal Intensive Care Units using the Evidence-based Practice for Improving Quality (REIN-EPIQ): a Study Protocol. Chin J Evid Based Pediatr 2018;13:452-7.

9. Zhu L, Zhang R, Zhang S, et al. Chinese neonatal birth weight curve for different gestational age. Zhonghua Er Ke Za Zhi 2015;53:97-103.

10. Lee SK, Aziz K, Dunn M, et al. Transport Risk Index of Physiologic Stability, version II (TRIPS-II): a simple and practical neonatal illness severity score. Am J Perinatol 2013;30:395-400.

11. Jobe AH, Bancalari E. Bronchopulmonary dysplasia. Am J Respir Crit Care Med 2001;163:1723-9.

12. Bell MJ, Ternberg JL, Feigin RD, et al. Neonatal necrotizing enterocolitis. Therapeutic decisions based upon clinical staging. Ann Surg 1978;187:1-7.

13. Walsh MC, Kliegman RM. Necrotizing enterocolitis: treatment based on staging criteria. Pediatr Clin North Am 1986;33:179-201.

14. Chinese Pediatric Society. Chinese Consensus of Diagnosis and Treatment of Neonatal Sepsis. Chin J Peditr 2003;41:897-9.

15. Stoll BJ, Hansen N, Fanaroff AA, et al. Late-onset sepsis in very low birth weight neonates: the experience of the NICHD Neonatal Research Network. Pediatrics 2002;110:285-91.

16. Papile LA, Burstein J, Burstein R, et al. Incidence and evolution of subependymal and intraventricular hemorrhage: a study of infants with birth weights less than 1,500 gm. J Pediatr 1978;92:529-34.

17. International Committee for the Classification of Retinopathy of Prematurity. The International Classification of Retinopathy of Prematurity revisited. Arch Ophthalmol 2005;123:991-9.

18. Qiu X, Lodha A, Shah PS, et al. Neonatal outcomes of small for gestational age preterm infants in Canada. Am J Perinatol 2012;29:87-94.

19. Ludvigsson JF, Lu D, Hammarström L, et al. Small for gestational age and risk of childhood mortality: A Swedish population study. PLoS Med 2018;15:e1002717.

20. Nobile S, Marchionni P, Carnielli VP. Neonatal outcome 
of small for gestational age preterm infants. Eur J Pediatr 2017;176:1083-8.

21. Bartels DB, Kreienbrock L, Dammann O, et al. Population based study on the outcome of small for gestational age newborns. Arch Dis Child Fetal Neonatal Ed 2005;90:F53-9.

22. Peacock JL, Lo JW, D'Costa W, et al.Respiratory morbidity at follow-up of small-for-gestational-age infants born very prematurely. Pediatr Res 2013;73:457-63.

23. Tul N, Lasic M, Bricelj K, et al. Outcome of small for gestational age preterm singletons: a population-based cohort study. J Perinat Med 2016;44:941-4.

24. Wignarajah D, Cock ML, Pinkerton KE, et al. Influence of intrauterine growth restriction on airway development in fetal and postnatal sheep. Pediatr Res 2002;51:681-8.

25. Orgeig S, Crittenden TA, Marchant C, et al. Intrauterine

Cite this article as: Huang Y, Zhang L, Sun H, Liu C, Yang Y, Lee SK, Cao Y, Jiang S; on behalf of the Reduction of Infection in Neonatal Intensive Care Units using the Evidence-based Practice for Improving Quality (REIN-EPIQ) Study Group. Neonatal outcome of small for gestational age infants born at 26-33 weeks' gestation in Chinese neonatal intensive care units. Transl Pediatr 2021;10(4):754-764. doi: 10.21037/tp-20-339 growth restriction delays surfactant protein maturation in the sheep fetus. Am J Physiol Lung Cell Mol Physiol 2010;298:L575-83.

26. Chan-Ling T, Gole GA, Quinn GE, et al. Pathophysiology, screening and treatment of ROP: A multi-disciplinary perspective. Prog Retin Eye Res 2018;62:77-119.

27. Ree IM, Smits-Wintjens VE, Rijntjes-Jacobs EG, et al. Necrotizing enterocolitis in small-for-gestational-age neonates: a matched case-control study. Neonatology 2014;105:74-8.

28. Fishel Bartal M, Chen HY, Blackwell SC, et al. Neonatal morbidity in late preterm small for gestational age neonates. J Matern Fetal Neonatal Med 2019:1-6.

29. Doron MW, Makhlouf RA, Katz VL, et al. Increased incidence of sepsis at birth in neutropenic infants of mothers with preeclampsia. J Pediatr 1994;125:452-8. 